

\title{
Mejoramiento a la gestión de calidad en la logística de aprovisionamiento. Caso de estudio: empresa pesquera acuícola
}

\author{
Dariel Rivadeneira Casanueva ${ }^{1^{*}} \mathbb{D}$, Orlando de la Cruz Rivadeneira ${ }^{2}(\mathbb{D}$, Damaris Taydi \\ Castillo Jiménez $^{1}{ }^{\mathbb{D}}$, Higinia Bismayda Gómez Avilés ${ }^{\mathbb{D}}$, Arelys López Concepción ${ }^{1} \mathbb{D}^{\mathbb{D}}$, \\ Alain Ulloa Zaila1 ${ }^{(\mathbb{D})}$
}

${ }^{1}$ Dpto. de Ingeniería Industrial, Facultad de Ciencias Técnicas y Empresariales, Universidad de Sancti Spíritus "José Martí Pérez", C.P. 60100, Cuba.

${ }^{2}$ Empresa Pesquera de Sancti Spíritus “PESCASPIR”, C.P. 60100, Cuba.

${ }^{3}$ Centro de Estudios de Energía de Procesos Industriales, Universidad de Sancti Spíritus “José Martí Pérez", C.P. 60100, Cuba.

*Autor de correspondencia: darielrc@uniss.edu.cu https://doi.org/10.22209/rt.v44n1a07

Recepción: 01 de julio de 2020 | Aceptación: 20 de octubre de 2020| Publicación: 01 de enero de 2021

\section{Resumen}

La investigación se realizó en una empresa pesquera de Sancti Spíritus, con el propósito de mejorar la calidad en el sistema logístico de aprovisionamiento de productos acuícolas, mediante modelos predictivos de calidad. La propuesta realizada permitió, con su aplicación parcial, prever la durabilidad de los productos pesqueros en función del tiempo y la temperatura de almacenamiento en el sistema logístico de aprovisionamiento. En el transcurso de la investigación y en el diseño del procedimiento, se emplearon diferentes métodos y técnicas: diagrama de flujo de actividades, diagrama de causa-efecto, diagrama de Pareto, la tormenta de ideas, método Delphi, diseño de experimento, método del índice de calidad, encuestas, consulta de documentos, entre otros. Se obtuvo como resultado la relación entre las curvas de deterioro y el método del índice de calidad de los diferentes tratamientos, lo que permitió obtener el modelo predictivo de calidad de los tratamientos 1, 2, 7 y 8, creándose las condiciones para la toma de decisiones, en la evaluación de la calidad del pescado fresco acuícola en la logística de aprovisionamiento de empresas pesqueras.

Palabras clave: mejoramiento de la calidad; modelos predictivos; sistema logístico; diseño de experimento; industrias pesqueras.

\section{Improvement to quality management in supply logistics. Case study: aquaculture fishing company}

\begin{abstract}
The research was carried out in a fishing company in Sancti Spíritus, with the aim of improving the quality of the logistics system for the supply of aquaculture products, using predictive quality models. The proposal made it possible, with its partial implementation, to provide for the durability of fish products as a function of time and temperature of storage in the logistical supply system. In the course of the research and in designing the procedure, different methods and techniques were used: activity flow diagram, cause-effect diagram, Pareto chart, brainstorming, Delphi method, experiment design, quality index method, surveys, document consultation, among others. As a result, the relationship between the deterioration curves and the quality index method of the different treatments was obtained, which made it possible to obtain the predictive quality model of treatments 1,2, 7, and 8, creating the conditions for decision-making in the evaluation of the quality of fresh aquaculture fish in the supply logistics of fishing companies.
\end{abstract}

Keywords: quality improvement; predictive models; logistics system; design of experiment; fishing industries. 


\section{Introducción}

El pescado es uno de los alimentos de origen animal más completos, por la cantidad y calidad de nutrientes que aporta al organismo humano, indispensable para una dieta equilibrada y saludable debido principalmente a su aporte valioso en proteínas de alto valor biológico (15 al 24\%), al contener aminoácidos esenciales para la vida, como metionina, cisteína, treonina, lisina (imprescindible para el crecimiento de los niños) y triptófano (imprescindible para la formación de la sangre); así como a su rico contenido $(0,1$ al $15 \%)$ en ácidos grasos poliinsaturados Omega-3, ácido docosahexaenoico y ácido eicosapentaenoico. Además de esto, destacan sus cantidades variables de vitaminas hidrosolubles, como B1, B2, B3; y liposolubles como la E. Aportan potasio, fierro, calcio (espinas); y en menores proporciones yodo, magnesio, fósforo y zinc. Para consolidarse así, como un alimento cuyo beneficio para la salud del consumidor cada vez es más patente [1].

Por otro lado, el pescado es uno de los productos más frágiles y perecederos que existen, debido a su gran contenido en determinados constituyentes como el agua, con una media de $77,2 \%$, aminoácidos libres, lípidos con alto grado de insaturación, compuestos nitrogenados no proteicos, enzimas autolíticas, etc.; que facilitan la puesta en marcha de una serie de vías de alteración, bien mediante alteraciones de origen endógeno, debido a la actividad de enzimas lipasas que actúan sobre las grasas; o bien por alteraciones de origen exógeno donde participan activamente las bacterias, efectuando procesos de degradación de aminoácidos y óxidos de aminas [1].

Los alimentos perecederos son aquellos con probabilidad de estropearse, descomponerse o que se vuelven inseguros para el consumo [2]. Tienen un tiempo de vida limitado que depende de las características del producto, de las condiciones de almacenamiento en las que se mantiene el producto y del tiempo, lo que ha dado lugar a una inmensa pérdida y desperdicio de alimentos, por lo que deben venderse a los consumidores dentro de la vida útil para garantizar la calidad y la seguridad, al tiempo que maximizan los beneficios [3].

Las principales causas operativas de desperdicios de pescado son las ineficiencias en la producción, el almacenamiento, la manipulación y el transporte [4]. Por lo tanto, la manipulación, conservación, almacenamiento, medidas posteriores a la cosecha y el transporte de los alimentos perecederos requieren una atención especial con el fin de mantener la calidad y atributos nutricionales, evitando el desperdicio y las pérdidas [5].

La Organización de las Naciones Unidas para la Alimentación y la Agricultura, ha estimado que las pérdidas pos-cosecha (debido al deterioro) continúan siendo el $25 \%$ de las capturas totales. Por lo tanto, la mejor utilización de los recursos acuáticos debe ser dirigida, sobre todo, a la reducción de estas enormes pérdidas, la preservación del pescado y de los productos pesqueros, así como el mejoramiento de la calidad en el sistema logístico de aprovisionamiento [2].

Los esfuerzos recientes se centran en los cambios de calidad y en la predicción de la vida útil del pescado. Por lo tanto, el uso de modelos para predecir los cambios de calidad es de considerable interés [6].

Emanado de la investigación realizada con precedencia y la consulta bibliográfica [7,8], las principales pérdidas en las características de calidad de los productos derivados de la pesca acuícola, se presentan en el sistema logístico de aprovisionamiento; donde el tiempo de vida depende de las características del producto, de las condiciones de almacenamiento en las que se mantiene el producto y del tiempo; ni un modelo predictivo de los cambios de calidad de la carpa común, basado en el tiempo y la temperatura de almacenamiento en el contexto cubano. A partir de esta necesidad, se reconoce que las prácticas actuales para la evaluación de la calidad de la carpa común en la logística de aprovisionamiento, no permite la toma de decisiones oportunas, lo que provoca pérdidas poscosechas, constituyendo este el problema a resolver. Como consecuencia, el objetivo de esta investigación se enmarca en el diseño de un procedimiento para la obtención de un modelo predictivo de calidad, que prevea la durabilidad de los productos pesqueros, en función del tiempo y la temperatura de almacenamiento, para la toma de decisiones oportunas que permitan reducir las pérdidas pos-cosechas.

\section{Materiales y métodos}

A partir de la revisión bibliográfica realizada y la situación problemática, se dio respuesta al problema de investigación, mediante el diseño de un procedimiento para la obtención de un modelo predictivo de calidad, basado en el análisis microbiano, bases volátiles totales nitrogenadas (BVTN) y análisis sensorial; en la gestión logística de aprovisionamiento a la industria pesquera acuícola. El procedimiento propuesto en la Figura 1 transita por 8 etapas, se aplica parcialmente hasta la etapa 3.

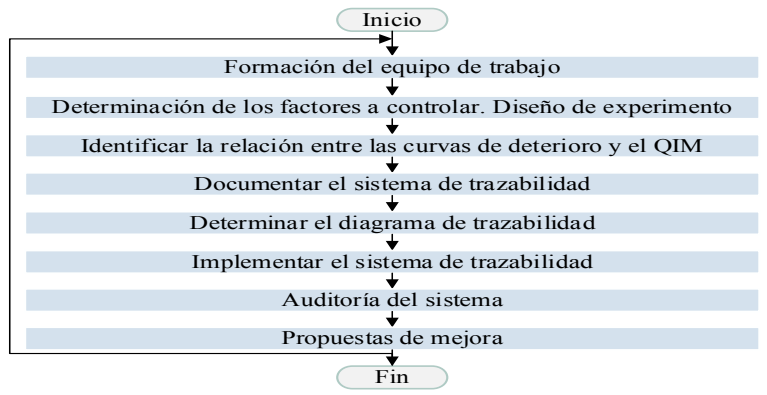

Figura 1. Etapas del procedimiento para el diseño de un modelo predictivo de calidad en la logística de aprovisionamiento de la empresa pesquera acuícola. QIM: método del índice de calidad. 
Comienza con la etapa 1, para la ejecución de este paso se recomienda el procedimiento aprobado en la NC 49:1981[9], y a que la cantidad de expertos depende de la complejidad y las características del trabajo a realizar. El grupo de expertos debe estar entre 7 y 15 para mantener un nivel de confianza y calificación elevado [9]. Para la determinación de la cantidad de expertos se utilizan criterios probabilísticos y se asume una distribución binomial [10]. Con este fin se utiliza la Ecuación1:

$$
M=\frac{p(1-p) k}{i^{2}}
$$

Donde M: cantidad de expertos; i: nivel de precisión deseado; p: proporción estimada de errores de los expertos; k: constante cuyo valor está asociado al nivel de confianza elegido.

En la etapa 2, se determinan los factores que inciden en la frescura del pescado y su durabilidad a través de métodos y herramientas a aplicar, tales como: tormenta de ideas, el método Delphi y el método de expertos basado en el cálculo del coeficiente de Kendall, y se aplicó el procedimiento del diseño de experimento estadístico (DEE),según Gutiérrez y de la Vara[11], el cual requiere considerar los pasos que se comentarán a continuación:

1. Determinar el problema de calidad.

2. Determinar factores a estudiar o investigar.

3. Elegir las variables de respuesta que serán medidas.

4. Seleccionar el diseño experimental adecuado a los factores que se tienen y al objetivo del experimento.

5. Planear y organizar el trabajo experimental.

6. Realizar el experimento.

7. Análisis estadístico.

8. Interpretación

9. Conclusiones finales.

Los resultados experimentales permitirán después calibrar y respaldar modelos matemáticos o computacionales que guarden relación con el fenómeno investigado [12].

En la etapa 3, para determinar la relación entre las curvas de deterioro y el método del índice de calidad (QIM, según sus siglas en inglés), se representó en forma tabla el grado de asociación lógica entre las variables a medir a partir de la recogida de datos, y el análisis posterior necesario para la confirmación de la correlación de la tabla.
Para documentar el sistema de trazabilidad en la etapa 4, se diseñaron las Tablas 1, 2, 3 y 4,para la recolección de datos:

Tabla 1. Codificación para la identificación de la materia prima en función de la procedencia.

\begin{tabular}{cccccc}
\hline Procedencia & $\begin{array}{c}\text { Punto de } \\
\text { pesca }\end{array}$ & $\begin{array}{c}\text { Brigada } \\
\text { de pesca }\end{array}$ & $\begin{array}{c}\text { Código (materia } \\
\text { prima) }\end{array}$ \\
\hline Presa X & $\mathrm{X}_{1}, \mathrm{X}_{2} \ldots, \mathrm{X}_{\mathrm{n}}$ & Brigada $\mathrm{Y}$ & $01: \mathrm{X}$ & $01: \mathrm{X}_{1}$ & $01: \mathrm{Y}$ \\
Presa X & $\mathrm{X}_{1}, \mathrm{X}_{2} \ldots, \mathrm{X}_{\mathrm{n}}$ & Brigada $\mathrm{Z}$ & $01: \mathrm{X}$ & $02: \mathrm{X}_{2}$ & $02: \mathrm{Z}$ \\
Presa A & $\mathrm{A}_{1}, \mathrm{~A}_{2} \ldots, \mathrm{A}_{\mathrm{n}}$ & Brigada $\mathrm{Y}$ & $01: \mathrm{A}$ & $01: \mathrm{A}_{1}$ & $01: \mathrm{Y}$ \\
Presa A & $\mathrm{A}_{1,} \mathrm{~A}_{2, \ldots, \mathrm{A}_{\mathrm{n}}}$ & Brigada Z & $01: \mathrm{A}$ & $01: \mathrm{A}_{1}$ & $02: \mathrm{Z}$ \\
\hline
\end{tabular}

Tabla 2. Etiqueta para la identificación de los lotes de pescado.

\begin{tabular}{|c|c|c|c|c|}
\hline \multicolumn{5}{|c|}{ Logotipo de la empresa } \\
\hline Especie & Tenca & Carpa & Tilapia & Claria \\
\hline \multicolumn{5}{|l|}{ Hora de captura } \\
\hline \multicolumn{5}{|c|}{$\begin{array}{l}\text { Hora de recepción } \\
\text { punto acopio }\end{array}$} \\
\hline \multicolumn{5}{|c|}{$\begin{array}{l}\text { Hora de recepción } \\
\text { industria }\end{array}$} \\
\hline Número de lote & L- & & & \\
\hline
\end{tabular}

Tabla 3. Parámetros a registrar en la identificación del pescado.

\begin{tabular}{ll}
\hline Parámetro & Utilidad \\
\hline $\begin{array}{l}\text { Nombre del } \\
\text { pescado }\end{array}$ & Identificación de la especie \\
Procedencia & $\begin{array}{l}\text { Identificación del embalse donde se } \\
\text { capturó (origen del pescado) } \\
\text { Identificación de la brigada de pesca } \\
\text { que realizó la captura. Delimitación de } \\
\text { responsabilidades }\end{array}$ \\
Método de captura & $\begin{array}{l}\text { Arte de pesca usado (métodos y estilos de } \\
\text { trabajo) }\end{array}$ \\
$\begin{array}{l}\text { Conocer el tiempo exacto que transcurrió } \\
\text { desde la captura hasta la recepción en el } \\
\text { exposición }\end{array}$ & $\begin{array}{l}\text { punto de acopio en el establecimiento } \\
\text { pesquero acuícola y luego hasta la } \\
\text { recepción en la industria pesquera } \\
\text { acuícola }\end{array}$ \\
$\begin{array}{l}\text { Enunciar las características que afectan } \\
\text { la calidad e inocuidad del pescado. } \\
\text { Determinar el nivel de deterioro de la } \\
\text { mescado }\end{array}$ & \begin{tabular}{l} 
materia prima \\
\hline
\end{tabular} \\
\hline
\end{tabular}


Tabla 4. Modelo de control para la identificación del pescado.

\begin{tabular}{|c|c|c|c|c|c|c|}
\hline \multicolumn{4}{|c|}{ Modelo de control para la identificación del pescado } & \multicolumn{3}{|c|}{ Mes: } \\
\hline \multicolumn{3}{|c|}{ Procedencia: } & \multirow{2}{*}{$\begin{array}{l}\text { Brigada de Pesca: } \\
\text { Hora llegada punto de acopio }\end{array}$} & Método c & aptura: & \\
\hline Fecha & Especie & $\begin{array}{l}\text { Hora de } \\
\text { captura }\end{array}$ & & $\begin{array}{l}\text { Hora } \\
\text { llegada } \\
\text { a la } \\
\text { industria }\end{array}$ & $\begin{array}{l}\text { Valor por demérito del } \\
\text { punto acopio }\end{array}$ & $\begin{array}{l}\# \text { de } \\
\text { lote }\end{array}$ \\
\hline
\end{tabular}

Para la determinación del diagrama de trazabilidad en la etapa 5, en el caso de la logística de aprovisionamiento a la industria pesquera acuícola, la trazabilidad es interna y se facilitará por medio del seguimiento de los lotes asociados a las brigadas de pesca identificadas por claves, así, se siguió la lógica en la que se basa la revisión de los procesos de trazabilidad, mediante el cumplimiento de los tres principios: identificación, registro y transmisión.

Una vez identificados los datos de la materia prima, en la etapa 6, se garantizará la toma de decisiones oportunas enfocadas hacia dos aspectos fundamentales: jerarquizar la entrada de la materia prima al proceso industrial; y determinar qué surtido hacer en dependencia del nivel de deterioro que tenga el pescado, lo que permitirá disminuir las pérdidas pos-cosechas.

En la etapa 7, se establecen comprobaciones sistemáticas que permiten evaluar el correcto funcionamiento del sistema y su eficacia, así como la detección de posibles mejoras.

En esta etapa 8, se propone la evaluación de la disminución de las pérdidas pos-cosechas, una vez implementado el sistema de trazabilidad en la logística de aprovisionamiento a la industria pesquera acuícola. Como herramienta fundamental pueden utilizarse los gráficos de tendencia y los gráficos de control por variables.

\section{Resultados y discusión}

Para la aplicación del procedimiento fueron abordadas cada una de las etapas que a él pertenecen, en aras de hacer lógica la secuencia de trabajo.

\section{Etapa 1. Formación del equipo de trabajo}

Se calculó el número de expertos mediante de la Ecuación1, donde $\mathrm{i}=0,1$; nivel de confianza $=99 \%$; $\mathrm{p}=0,01$; $\mathrm{k}=6,6564$; donde se obtuvo un total de siete expertos. A partir de este análisis, se seleccionaron aquellos con un coeficiente de competencia que estuvieron más próximo al valor 1 .

\section{Etapa 2. Determinación de los factores pre y pos cosecha a controlar. Diseño de experimento}

Una vez seleccionados los expertos, se aplicó una tormenta de ideas para identificar todos los factores pre y pos-cosecha que afectan la frescura del pescado. Luego, se procedió a utilizar el método Delphi por su adecuación en la validación de los factores establecidos como más apropiados, según las condiciones del medio de cultivo y las condiciones de la empresa. Para esto se le entregó a cada uno de los expertos seleccionados, un cuestionario para obtener criterios sobre el procedimiento a aplicar, y mediante una hoja de cálculo en Microsoft Excel, se procesó el criterio de los expertos seleccionados para validar el procedimiento. La misma quedó estructurada de la forma siguiente:

Usando una tabla se registraron los criterios de cada experto y se tomaron como variables, los criterios y las categorías de la escala como valores de las variables (muy adecuado $=1$, bastante adecuado $=2$, adecuado $=$ 3 , poco adecuado $=4$ e inadecuado $=5$ ); una tabla de frecuencia absoluta donde se tomaron como variables los aspectos y categorías de la escala como valores de las variables; una tabla de frecuencias acumuladas absolutas; una tabla de frecuencias acumuladas relativas; y una tabla que permitió determinar los puntos de corte y la escala de los aspectos considerados. La obtención de los puntos se obtuvo a través del cálculo de N-P, donde:

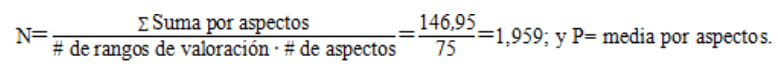

Al cumplirse la condición: N-P <punto de corte $(-1,047<-0,118)$, la opinión de los expertos es válida. En tal sentido, se llegó a la conclusión de que los factores pre y pos-cosecha que más afectan la calidad e inocuidad de la carpa, y que por lo tanto son necesarios tener en cuenta en cualquier estudio a realizar, son: especie, edad, peso, condiciones de producción, temperatura del agua, contaminación química, contaminación microbiana, malas prácticas de manipulación y transportación, fase tiempotemperatura, incumplimiento de las normas sanitarias y conservación de la cadena de frío.

\section{Aplicación del procedimiento diseño de experimento estadístico:}

1. En la empresa objeto de estudio no se manejan los registros de las pérdidas pos-cosecha ocasionado, por esta razón, se toman como referencia los estudios realizados en los países en vía de desarrollo. Se definen como indicadores para medir el éxito del proyecto de mejora, los siguientes: rendimiento industrial; relación tiempo-temperatura y utilización del hielo por toneladas capturadas. 
Tabla 5. Niveles de prueba para cada factor.

\begin{tabular}{|c|c|c|c|c|c|}
\hline \multicolumn{2}{|c|}{ Condiciones de producción } & \multicolumn{4}{|c|}{ Contaminación del embalse (CE) } \\
\hline \multirow{5}{*}{ 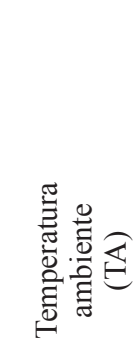 } & & \multirow{2}{*}{\multicolumn{2}{|c|}{$\begin{array}{l}\text { - Punto menos contaminado } \\
\text { Capacidad de nevado (CN) }\end{array}$}} & \multirow{2}{*}{\multicolumn{2}{|c|}{$\begin{array}{l}\text { + Punto más contaminado } \\
\text { Capacidad de nevado (CN) }\end{array}$}} \\
\hline & & & & & \\
\hline & \multirow[b]{2}{*}{ - Mañana } & + En la captura & - En tierra & + En la captura & - En tierra \\
\hline & & $\begin{array}{c}-\mathrm{TA}-\mathrm{CE}+\mathrm{CN} \\
\text { (1) }\end{array}$ & $\begin{array}{c}\text {-TA-CE-CN } \\
\text { (2) }\end{array}$ & $\begin{array}{c}-\mathrm{TA}+\mathrm{CE}+\mathrm{CN} \\
\text { (3) }\end{array}$ & $\begin{array}{c}-\mathrm{TA}+\mathrm{CE}-\mathrm{CN} \\
\text { (4) }\end{array}$ \\
\hline & + Tarde & $\begin{array}{l}+\mathrm{TA}-\mathrm{CE}+\mathrm{CN} \\
(5)\end{array}$ & $\begin{array}{l}+\mathrm{TA}-\mathrm{CE}-\mathrm{CN} \\
(6)\end{array}$ & $\begin{array}{c}+\mathrm{TA}+\mathrm{CE}+\mathrm{CN} \\
(7)\end{array}$ & $\begin{array}{c}+\mathrm{TA}+\mathrm{CE}-\mathrm{CN} \\
(8)\end{array}$ \\
\hline
\end{tabular}

Arte de pesca: bocana; especie: carpa común; edad: $2-3$ años y peso: $1,8-2,3 \mathrm{~kg}$

2. Dentro de los factores que influyen de manera significativa en la variable de respuesta se encuentran: especie, edad, peso, condiciones de producción y contaminación del embalse, que se consideran variables de entrada. Los niveles de prueba para cada factor, se muestran en la Tabla 5.

3. Las características de calidad que se derivan del análisis microbiológico y sensorial en las que se espera se reflejen los cambios producidos en los factores controlables y no controlables, y que se pueden medir de manera confiable, son: Escherichia coli, colonias de microorganismos, coliformes, coliformes termotolerantes, piel, rigidez, ojos y branquias.

4. Se selecciona el diseño factorial $2^{\mathrm{k}}(\mathrm{k}$ factores con dos niveles de prueba cada uno) y dentro de este el modelo $2^{3}$, por ser tres factores los que inciden en la variable respuesta, y por tanto, son necesarios estudiar $(2 \leq \mathrm{k}$ $\leq 5)$. Consta de $2^{3}=8$ tratamientos diferentes.

La estimación del número de pescados a correr en cada tratamiento, se calculó con la siguiente Ecuación 2:

$$
\mathrm{m}=(2,5)^{2}\left(\frac{1-\mathrm{p}_{0}}{\mathrm{p}_{0}}\right)
$$

Donde $\mathrm{p}_{0}$ es la proporción utilizada como base y $(2,5)^{2}$ una constante; en este experimento $\mathrm{p}_{0}=0,6$ y cuando se sustituye en la Ecuación 2, se obtiene que $\mathrm{m}=5$ pescados por tratamiento, para poder detectar los efectos que tiene en cada factor de manera satisfactoria

Con el diseño factorial completo $2^{3}$ se pueden estudiar en total los $2^{\mathrm{k}-1}$ efectos, como muestra la Ecuación 3:

$$
\left(\begin{array}{l}
\mathrm{k} \\
\mathrm{r}
\end{array}\right)=\frac{\mathrm{k} !}{\mathrm{r} !(\mathrm{k}-\mathrm{r}) !}
$$

Con esta Ecuación 3, se obtienen como resultados, 3 efectos principales, 3 interacciones dobles y 1 interacción triple, lo que da un total de 7 efectos.
5. Se trabajó con: el grupo de expertos seleccionados, las muestras de pescado capturadas en la presa Zaza perteneciente a la empresa pesquera "PESCASPIR" en los horarios de la mañana y la tarde, en el punto más y menos contaminado del embalse y se nevó una parte en la embarcación y el resto cuando llegó a tierra. Luego, se trasladaron dichas muestras hasta la empresa pesquera industrial de Sancti Spíritus "EPISAN", donde se realizaron las pruebas correspondientes.

6. En esta fase experimental, se determinó el límite máximo de microorganismos a $30^{\circ} \mathrm{C}$ que puede presentar la carpa común para considerarse inocua para el consumo humano (hasta $1,0 \times 10^{5}$ unidades formadoras de colonias por gramo, de acuerdo a la NC 585:2017 [13]). Los coliformes a $45{ }^{\circ} \mathrm{C}$ en la carpa común, se consideran inocuos mientras su valor sea cero, lo cual significa que, a partir de la presencia de estas bacterias en la especie, se encuentra deteriorada. Como se determinó la presencia de coliformes a $45{ }^{\circ} \mathrm{C}$, del tratamiento 1 al noveno día y en el tratamiento 2 al séptimo día, los valores del crecimiento microbiano se mantuvieron iguales hasta ese día; para el tratamiento 7 y 8 se mantuvo inocuo hasta el cuarto y quinto día, respectivamente (Tabla 6).

Tabla 6. Análisis microbiológico al parámetro de calidad crecimiento microbiano de la carpa común capturada en el turno de las 10:00 am (Número de bacterias-gramo).

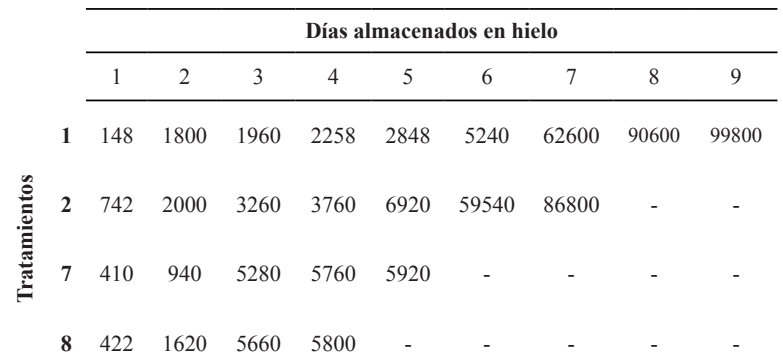


Con los datos obtenidos en las etapas anteriores y mediante el QIM (Tabla 7), se llevaron a cabo los análisis sensoriales necesarios para detectar el deterioro de la carpa común hasta el momento que no se considera inocua para la salud humana. El análisis sensorial general con una puntuación por deméritos, donde la suma total oscila entre 0 (frescura total) y 22 (pérdida total de frescura), demostró que la carpa común en las condiciones ideales puede alcanzar hasta nueve días de almacenamiento en hielo en el horario de la mañana, mientras que en condiciones desfavorables solo hasta siete días en el mismo horario (Tabla 8).

Tabla 7. Parámetros de calidad para medir indicador de frescura en el pescado.

\begin{tabular}{lcc}
\hline \multicolumn{1}{c}{ Parámetros de calidad } & Puntuaciones \\
\hline \multirow{3}{*}{ Piel } & Apariencia/color & $0=-2$ \\
& Mucus & $0=-2$ \\
Rigidez/textura & Olor & $0=-=3$ \\
Ojos & Cornea & $0=3$ \\
& Pupila & $0=-=3$ \\
\multirow{2}{*}{ Branquias o agallas } & $0=2$ \\
& Adherencia & $0=3$ \\
& Color & $0=2$ \\
& Olor & $0=2$ \\
\multicolumn{2}{c}{ Índice de calidad } & $\mathbf{0}=\mathbf{2 2}$ \\
\hline
\end{tabular}

Tabla 8. Valores por deméritos del análisis sensorial al parámetro de calidad frescura de la carpa común capturada en el turno de las 10:00 am.

\begin{tabular}{|c|c|c|c|c|c|c|c|c|c|c|}
\hline & & \multicolumn{9}{|c|}{ Días almacenados en hielo } \\
\hline & & 1 & 2 & 3 & 4 & 5 & 6 & 7 & 8 & 9 \\
\hline \multirow{8}{*}{ 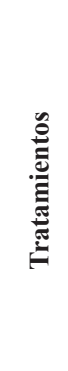 } & 1 & 0 & 0 & 1 & 2 & 3 & 4 & 8 & 14 & 22 \\
\hline & 2 & 0 & 2 & 3 & 3 & 7 & 12 & 19 & 22 & - \\
\hline & 3 & 0 & 0 & 1 & 8 & 9 & 15 & 20 & 22 & - \\
\hline & 4 & 0 & 0 & 6 & 10 & 16 & 19 & 22 & - & - \\
\hline & 5 & 0 & 1 & 2 & 3 & 3 & 10 & 15 & 22 & - \\
\hline & 6 & 0 & 2 & 3 & 5 & 11 & 18 & 22 & - & - \\
\hline & 7 & 0 & 0 & 4 & 9 & 13 & 17 & 22 & - & - \\
\hline & 8 & 0 & 1 & 8 & 10 & 15 & 21 & 22 & - & - \\
\hline
\end{tabular}

7. Se realizó un análisis de varianza (ANOVA, Tablas 9 y 10), para todos los parámetros microbiológicos y sensoriales, con intervalos de confianza simultáneos y prueba de Tukey al 99\% y un nivel de confianza individual de 99,96\%.
Tabla 9. ANOVA: análisis microbiológico general vs. días.

\begin{tabular}{cccccc}
\hline Fuente & $\begin{array}{c}\text { Grados de } \\
\text { libertad }\end{array}$ & $\begin{array}{c}\text { Suma de } \\
\text { cuadrados }\end{array}$ & $\begin{array}{c}\text { Cuadrado } \\
\text { medio }\end{array}$ & $\begin{array}{c}\text { Valor } \\
\mathbf{F}\end{array}$ & $\begin{array}{c}\text { Valor } \\
\mathbf{p}\end{array}$ \\
\hline Día & 8 & 23121215834 & 2890151979 & 25,76 & 0,000 \\
Error & 16 & 1794788097 & 112174256 & - & - \\
Total & 24 & 24916003930 & - & - & - \\
& $\mathrm{S}=10591$ & $\mathrm{R}^{2}=92,80 \%$ & $\mathrm{R}^{2}$ (ajustado)=89,19\% & \\
\hline
\end{tabular}

F: variación entre las medias de las muestras/variación dentro de las muestras y p: proporción.

Tabla 10. ANOVA: análisis sensorial general vs. días.

\begin{tabular}{cccccc}
\hline Fuente & $\begin{array}{c}\text { Grados de } \\
\text { libertad }\end{array}$ & $\begin{array}{c}\text { Suma de } \\
\text { cuadrados }\end{array}$ & $\begin{array}{c}\text { Cuadrado } \\
\text { medio }\end{array}$ & Valor F & Valor p \\
\hline Día & 8 & 3147,1 & 393,4 & 27,48 & 0,000 \\
Error & 52 & 744,4 & 14,3 & - & - \\
Total & 60 & 3891,4 & - & - & - \\
& $\mathrm{S}=3,784$ & $\mathrm{R}^{2}=80,87 \%$ & $\mathrm{R}^{2}$ (ajustado) $=77,93 \%$ & \\
\hline
\end{tabular}

F: variación entre las medias de las muestras/variación dentro de las muestras y p: proporción.

Se comprueba que existen diferencias significativas bajo las condiciones más o menos favorables del diseño estadístico experimental, para todos los parámetros microbiológicos y sensoriales $(\mathrm{p}=$ $0,000, p>0,01)$, lo que demuestra que la contaminación del embalse, la temperatura ambiente y la capacidad de nevado, influyen en la toma de decisiones oportunas para el procesamiento industrial de la carpa común.

8. En el análisis microbiológico, solo se llevaron a cabo las curvas de deterioro de los tratamientos 1, 2, 7 y 8 . Los demás tratamientos no se hicieron por el costo de los experimentos. En la Figura 2 se muestra el análisis microbiológico, en cual se determinó que la especie carpa común en las condiciones ideales de prey pos-cosecha tiene una duración total de nueve días de almacenamiento en hielo en el horario de las 10:00 am, en caso contrario se considera inocua para el consumo humano hasta el cuarto día a las 10:00am.

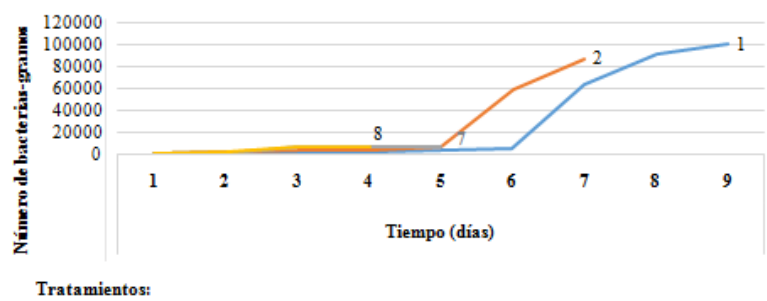

Figura 2. Análisis de la vida útil de la carpa común en relación con el número de bacterias-gramos. 
En el análisis sensorial se llevaron a cabo las curvas de deterioro de los 8 tratamientos, como se muestra en la Figura 3. En este análisis, se determinó que en las condiciones ideales de pre y poscosecha tiene una duración total de nueve días de almacenamiento constante en hielo, mientras que en las condiciones más desfavorables se considera inocua para el consumo humano hasta el séptimo día.

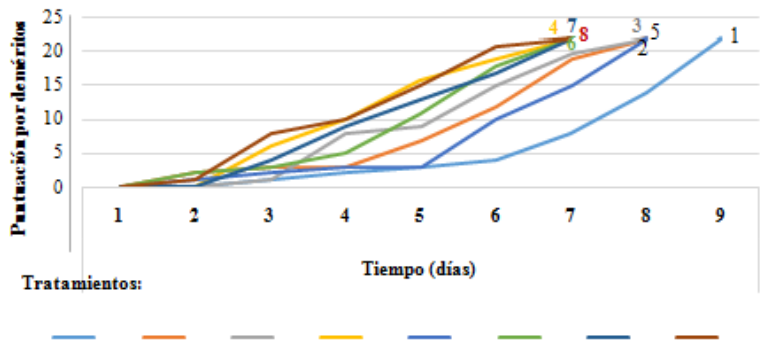

Figura 3. Análisis de la vida útil de la carpa común de acuerdo a la puntuación por deméritos en la Tabla 7.
9. De acuerdo con los análisis realizados, se confirma que la carpa común en relación con el crecimiento microbiano y la puntuación por demérito, tiene una duración máxima en las condiciones óptimas de manipulación, almacenamiento y transportación del medio de cultivo, entre otras, de 9 días; por lo que para generalizar el resultado del diseño experimental, y de esta forma lograr que las mejoras se mantengan, se deben seguir las normas higiénicas sanitarias correctas de manipulación, conservar la cadena de frío desde la captura y en el caso de no tener las condiciones ideales para esto, nevar al llegar la embarcación a tierra. Siempre se debe tener en cuenta el horario en que se realizan las capturas, los puntos de pesca, la edad, peso de la carpa y el arte de pesca.

Etapa 3. Determinar la relación entre las curvas de deterioro y el método del índice de calidad

En esta etapa se determinó la relación existente entre las curvas de deterioro y el QIM, donde se tomaron en cuenta solo los límites máximos y mínimos de vida útil, los cuales se muestran en la Tabla 11.

Tabla 11. Relación entre las curvas de deterioro y el método del índice de calidad de los límites máximos y mínimos de vida útil de los tratamientos 1, 2, 7 y 8.

\begin{tabular}{|c|c|c|c|c|c|c|c|c|c|c|c|}
\hline \multirow{6}{*}{$\begin{array}{l}\text { Límite } \\
\text { máximos } \\
\text { de vida útil }\end{array}$} & \multirow{3}{*}{$\begin{array}{l}\text { Tratamiento } 1 \\
(-\mathrm{TA}-\mathrm{CE}+\mathrm{CN})\end{array}$} & Día & 1 & 2 & 3 & 4 & 5 & 6 & 7 & 8 & 9 \\
\hline & & $\begin{array}{c}\text { Puntuación por } \\
\text { deméritos }\end{array}$ & 0 & 0 & 1 & 2 & 3 & 4 & 8 & 14 & 22 \\
\hline & & $\begin{array}{l}\text { Crecimiento } \\
\text { microbiano }\end{array}$ & 148 & 1800 & 1960 & 2258 & 2848 & 5240 & 62600 & 90600 & 99800 \\
\hline & \multirow{3}{*}{$\begin{array}{l}\text { Tratamiento } 2 \\
(-\mathrm{TA}-\mathrm{CE}-\mathrm{CN})\end{array}$} & Día & 1 & 2 & 3 & 4 & 5 & 6 & 7 & 8 & - \\
\hline & & $\begin{array}{c}\text { Puntuación por } \\
\text { deméritos }\end{array}$ & 0 & 2 & 3 & 3 & 7 & 12 & 19 & 22 & - \\
\hline & & $\begin{array}{l}\text { Crecimiento } \\
\text { microbiano }\end{array}$ & 742 & 2000 & 3260 & 3760 & 6920 & 59540 & 86800 & - & - \\
\hline \multirow{6}{*}{$\begin{array}{l}\text { Límite } \\
\text { mínimo de } \\
\text { vida útil }\end{array}$} & \multirow{3}{*}{$\begin{array}{c}\text { Tratamiento } 7 \\
(+\mathrm{TA}+\mathrm{CE}+\mathrm{CN})\end{array}$} & Día & 1 & 2 & 3 & 4 & 5 & 6 & 7 & - & - \\
\hline & & $\begin{array}{c}\text { Puntuación por } \\
\text { deméritos }\end{array}$ & 0 & 0 & 4 & 9 & 13 & 17 & 22 & - & - \\
\hline & & $\begin{array}{l}\text { Crecimiento } \\
\text { microbiano }\end{array}$ & 410 & 940 & 5280 & 5760 & 5920 & - & - & - & - \\
\hline & \multirow{3}{*}{$\begin{array}{l}\text { Tratamiento } 8 \\
(+\mathrm{TA}+\mathrm{CE}-\mathrm{CN})\end{array}$} & Día & 1 & 2 & 3 & 4 & 5 & 6 & 7 & - & - \\
\hline & & $\begin{array}{c}\text { Puntuación por } \\
\text { deméritos }\end{array}$ & 0 & 1 & 8 & 10 & 15 & 21 & 22 & - & - \\
\hline & & $\begin{array}{l}\text { Crecimiento } \\
\text { microbiano }\end{array}$ & 422 & 1620 & 5660 & 5800 & - & - & - & - & - \\
\hline
\end{tabular}


En la obtención del modelo predictivo (Tabla 11), como se puede observar el valor de la variable (crecimiento microbiano) aumenta con el valor de la variable (puntuación por deméritos) para cada uno de los tratamientos 1, 2, 7 y 8, lo que se clasifica como una correlación fuerte positiva.

\section{Conclusiones}

La aplicación parcial del procedimiento diseñado permitió identificar los factores pre y poscosecha, que más afectan la frescura del pescado (especie, edad, peso, condiciones de producción, temperatura del agua, contaminación del embalse, malas prácticas de manipulación y transportación, fase tiempo-temperatura, incumplimiento de las normas sanitarias y conservación de la cadena de frío). Se definieron las variables de entrada que influyen en las variables de respuesta (especie, edad, peso, condiciones de producción y la contaminación del embalse) las cuales fueron medidas con métodos microbiológicos y el método del índice de calidad (QIM). Se lograron identificar los diferentes tratamientos 1,2 , $3,4,5,6,7$ y 8 a estudiar en el diseño experimental $\left(2^{\mathrm{k}}=\right.$ $2^{3}=8$ ). En el procedimiento, se determinaron también que existen diferencias significativas entre las condiciones más favorables y menos favorables de los tratamientos identificados. A modo de conclusión del diseño de experimento estadístico, se comprobó que la carpa común con relación al crecimiento microbiano y la puntuación por demérito, tiene una duración máxima de 9 días, manteniendo las condiciones óptimas de manipulación, almacenamiento y transportación. Además, se determinó la relación entre las curvas de deterioro y el QIM de los diferentes tratamientos, lo que permitió obtener el modelo predictivo de calidad de los tratamientos $1,2,7$ y 8. De ese modo, las prácticas de evaluación de la calidad del pescado en el sistema logístico de aprovisionamiento, obtienen información más confiable sobre la durabilidad de los productos pesqueros y facilita la toma de decisiones oportunas en aras de reducir las pérdidas pos-cosecha.

\section{Referencias bibliográficas}

[1] Cabellos García J. M.: Aplicación de hidrocoloides, biopreservación, liofilización y radiación en conservación de pescado. Universidad Nacional de Trujillo, (2015).

[2] FAO: El estado mundial de la pesca y la acuicultura. Cumplir los objetivos de desarrollo sostenible. Roma, Italia, (2018).

[3] Xue M., Zhang J. y Tang W.: "Optimal temperature control for quality of perishable foods". ISATransactions. The Journal of Automation, Vol. 53, No. 2, (2014), 542-546.
[4] Lemma Y., Kitaw D. y Gatew G.: "Loss in Perishable Food Supply Chain: An Optimization Approach Literature Review". International Journal of Scientific \& Engineering Research,Vol. 16, No. 5, (2014).

[5] FAO: The State of World Fisheries and Aquaculture. Roma, Italia, 2016.

[6] Shi C., Lu H., Cui H. y Luo Y.: "Study on the predictive models of the quality of silver carp (Hypophthalmichthys molitrix) Fillets stored under variable temperature conditions". Journal of Food Processing and Preservation, Vol. 38, No. 1, (2014), 356-363.

[7] Martínez I.: "Problemas de codificación de productos que afectan la gestión de inventarios: Caso de estudio en empresas cubanas". DYNA, Vol. 81, No. 187, (2014), 64-72.

[8] Saslavsky D. y Shepherd B.: "Facilitating international production networks: The role of trade logistics". The Journal of International Trade \& Economic Development,Vol. 23, No. 7, (2014), 979-999.

[9] Oficina Nacional de Normalización: "Control de la calidad. Métodos de expertos”. NC 49:1981. Cuba, (1981).

[10] Lao León, Y. O., Pérez Pravia, M. C. y Delgado, F. M.: "Procedimiento para la selección de la Comunidad de Expertos con técnicas multicriterio". Ciencias Holguín, Vol. 22, No. 1, (2016), 34-49.

[11] Gutiérrez Pulido H. y de la Vara Salazar R.: Análisis y Diseño de Experimentos. México, McGraw-Hill, 2008.

[12] Mieles Bravo Y., Larrúa Quevedo R. y Alcivar Moreira S.: Diseño de experimento para vigas con nudos híbridos. Rev. Téc. Ing. Univ. Zulia, Vol. Especial, No. 2, (2020).

[13] Oficina Nacional de Normalización: "Contaminantes microbiológicos en alimentosRequisitos sanitarios”. NC 585:2017. Cuba, (2017). 


\section{REVISTA TECNICA}

DE LA FACULTAD DE INGENIERIA

UNIVERSIDAD DEL ZULIA

Vol. 44. N`1, Enero - Abril 2021, pp. 04 - 58

Esta revista fue editada en formato digital y publicada en Diciembre de 2020, por el Fondo Editorial Serbiluz, Universidad del Zulia. Maracaibo-Venezuela

www.luz.edu.ve

www.serbi.luz.edu.ve

www.produccioncientificaluz.org 